\title{
Hard Facts about Stones: Pulpal Calcifications: A Review
}

\author{
Nupura Aniket Vibhute ${ }^{1^{*}}$, Vibhute Aniket $\mathrm{H}^{2}$, Daule RajendraT ${ }^{3}$, Bansal Puja $\mathrm{P}^{4}$ and Mahalle Aditi ${ }^{5}$ \\ ${ }^{1}$ Department of Oropathology and Microbiology, Kims University, Hyderabad, India \\ ${ }^{2}$ Department of Orthodontia, Bharati Vidyapeeth Dental College And Hospital, Pune,Maharashtra, India \\ ${ }^{3}$ Department of Conservative Dentistry, Bharati Vidyapeeth Dental College And Hospital, Pune,Maharashtra, India \\ ${ }^{4}$ Department of Oral Pathology and Microbiology, Schiool of Dental Sciences, Sharda University, Greater Noida, India \\ ${ }^{5}$ Department of Oral Pathology and Microbiology, Dr. D. Y. Patil Dental College and Hospital, Pimpri, Pune, India
}

*Corresponding author: Vibhute NK, Department of Oropathology and Microbiology, Kims University, Hyderabad, India, Tel: 919637689097; E-mail: drnupura@gmail.com

Received date: Nov 13, 2015, Accepted date: Dec 30, 2015, Published date: Jan 7, 2016

Copyright: ( 2016 Vibhute NK, et al. This is an open-access article distributed under the terms of the Creative Commons Attribution License, which permits unrestricted use, distribution, and reproduction in any medium, provided the original author and source are credited.

\section{Abstract}

Pulp calcifications stones are discrete calcifications within the pulps of teeth. They are a frequent finding on bitewing and periapical radiographs. Johnson and Bevelander stated that a single tooth may have stones ranging from 1 to 12 or even more, with sizes varying from minute particle to large masses that occlude the pulp space. They often develop in teeth that appear quite normal in other respects. They have been seen in both functional as well as embedded teeth. Inspite of this frequent occurrence, they have received relatively little attention in textbooks. The purpose of this review was to examine these pulp stones from all the aspects including their pathogenesis, clinical aspects, histopathology, current perspectives and contemporary research on the same.

Keywords: Pulp stones; Denticles; Pulp calcifications

\section{Introduction}

Pulp calcifications stones are nodular, calcified masses appearing in either or both the coronal or root portions of the pulp organ. They often develop in teeth that appear quite normal in other respects. They have been seen in both functional as well as embedded teeth. They are usually asymptomatic unless they impinge on the nerves or blood vessels. Johnson and Bevelander [1] stated that a single tooth may have stones ranging from 1 to 12 or even more, with sizes varying from minute particle to large masses that occlude the pulp space. Despite a number of microscopic and histochemical studies, the exact cause of such pulp calcifications remains largely unknown. Thus, the purpose of this review is to examine these pulp stones from all the aspects including their pathogenesis, clinical aspects, histopathology, current perspectives and contemporary research on the same.

\section{Incidence}

A number of studies have been carried out to determine the actual incidence of pulp calcifications, and the results of these investigations are in essential agreement. For example Wilman [2] reported that of a series of 164 teeth picked at random and examined histologically, 143 (or $87 \%$ ) exhibited calcification ins the pulp. Interestingly only $15 \%$ of the areas of calcification were large enough to be seen on the dental radiograph. These findings confirm the investigations of Hill [3], who reported calcifications in $66 \%$ of all teeth examined in young persons between ages of 50 and 70 years. There is no apparent difference in the frequency of occurrence either between genders or among teeth in the dental arch.

According to one estimate, $66 \%$ of teeth in persons 10 to 30 years of age, $80 \%$ in those between 30 and 50 years and $90 \%$ in those over 50 years contain calcifications of some type [4].
In a radiographic assessment of the prevalence of pulp stones in a group of Turkish dental patients by Gulsahi et al., [5], it was found that as age increased, the prevalence of pulp stones increased $(\mathrm{P}<0.01)$. Molars had statistically more pulp stones than premolars and incisors $(\mathrm{P}<0.001)$.

Hamasha and Darwazeh [6] assumed that molars are the largest teeth in the arch and have a better supply of blood to pulp tissue, which may precipitate more calcification.

In the study by Nayak et al., [7], frequency of pulp stone on the right side was higher than left side, which is in contrast to findings by Ranjitkar [8]. Higher frequency of pulp stones were reported in maxillary arch compared to mandibular arch by Nayak et al., [7], but similar frequency in both arches [9] or higher occurrence in mandibular arch have also been reported [10].

Pulp stones extending to the entire dentition are infrequent. Radiographic diagnosis and a multidisciplinary approach are of paramount importance for successful treatment in cases of generalized pulp stones.

Donta et al., [11] presented a mildly mentally retarded patient with generalized pulp stone formation with a six-year follow-up in whom pulp stones were radiographically detected in the pulp chamber of all permanent teeth. They advocated that such patients should be referred for genetic evaluation because pulp stones are mostly associated with genetic dentine defects.

Ozkalayci et al., [12] presented a report of an unusual case of a 13year-old girl with generalized pulp stones with clinically normal crowns. Orthodontic treatment, gingivoplasty, and esthetic restorations were performed, thus using a multidisciplinary approach to establish functionally and esthetically sound dentition. 


\section{Etiology}

The etiology of various types of pulp calcifications is unknown. Although the incidence appears to increase with age of the persons, there is no definite association with pulpal irritation or inflammation such as that arising from caries or trauma. Since pulp calcifications have been reported from unerupted teeth, it is doubtful whether pulpal disease such as inflammation is of any significance.

Kretschmer and Seybold [13] reported that an extremely high percentage of pulp stones yield a pure streptococci upon culture. On this basis it has been suggested that microorganisms are the cause of pulp calcifications. Since the pulps of affected teeth were reportedly normal, aside from the calcification, and since it is well recognized that bacteria may be forced into the pulp tissue at the time of tooth extraction, it is most unlikely that bacteria are of any significance in the development of these pulp nodules.

Stafne and Szabo [14] attempted to correlate pulp nodules with various local or systemic diseases including cholelithiasis, renal lithiasis, arteriosclerosis, gout, acromegaly, osteitis deformans, hypercementosis, and torus mandibularis or palatinus. Their data indicates that no clear-cut relation exists between any of these conditions and pulp calcification.

Sundell et al., [15] and his associates attempted to determine whether the degree of pulp response elicited by cutting procedures and restorative materials was capable of increasing the incidence of pulp stone nidi and pulp stones. They found no significant correlation between pulp stones or nidi and the age and gender of the patient, the thickness of remaining dentin, beneath the cavity preparation, the preparation time, or the traumatic potential of the operative procedure. In addition, these workers proposed a combination of several hypotheses from literature including thrombosis or vascular wall injury or both, leading to pulp stone formation.

A theory has been advanced that the development of true denticle is caused by inclusion of remnant $s$ of epithelial root sheath within the pulp. These remnants induce the cells of the pulp to differentiate into odontoblasts, which form dentin masses called pulp stones [4].

\section{Types/Classification}

The two chief morphologic forms of pulp calcifications are discrete pulp stones (pulp nodules, denticles) and diffuse calcifications. Pulp stones are classified as per their structure into true denticles and false denticles.

True denticles are made up of localized masses of calcified tissue that resemble dentin because of their tubular nature. Actually these tubules bear greater resemblance to secondary dentin than to primary dentin, since the tubules are few and irregular. They are considerably more common in pulp chamber than in root canal [16].

True denticles may be further classified into free or attached. Denticles lying entirely within the pulp tissue and not attached to the dentinal walls are called free denticles, while those continuous with the dentinal wall are referred to as attached denticles.

Attached denticles are thought to be more common than the free denticles. However it should be remembered that though a denticle may appear free in one plane of section in which it is visualized it may be attached in another plane. Thus, without serial sections on an entire tooth pulp, one cannot state with any degree of assurance that a given denticle is free or attached.
False denticles are composed of localized masses of calcified material and unlike true denticles do not exhibit tubules. Instead the nodules appear to be made up of concentric lamellae or layers deposited around a central nidus. The exact nature of this nidus is unknown, although Johnson and Bevelander believe it is composed of cells, as yet unidentified, around which is laid down a layer of reticular fibers that subsequently calcify [16].

False denticles may be also further classified into free or attached. Sometimes as the concentric deposition of calcified material continues, it approximates and finally is in apposition with the dentinal wall. Here it may eventually become surrounded by secondary dentin, and is referred to as interstitial denticle. False denticles, which occur more commonly in the pulp chamber than in the root canal, are generally somewhat larger than true denticles. They may fill nearly the entire pulp than true denticles which are seldom larger than a fraction of a millimeter in diameter [16].

Johnson and Bevelander concluded from their studies that a differentiation between true and false denticles should not be drawn, since all denticles originally show no tubules even though subsequently may become surrounded by tissue containing dentinal tubules [1].

Diffuse Calcifications are most commonly seen in the root canals of teeth and resemble the calcification seen in other tissues of the body following degeneration. This type of calcification is frequently termed calcific degeneration. Its usual pattern is amorphous, unorganized linear strands or columns paralleling the blood vessels and nerves of pulp [16].

\section{Histopathology}

Denticles consist of tubular dentin surrounding a central nest of epithelium. With time, the central epithelium degenerates and the tubules undergo sclerosis, making their detection difficult [17].

Pulp stones demonstrate a central amorphous mass of irregular calcification surrounded by concentric lamellar rings of regular calcified material. Occasionally, a peripheral layer of tubular dentin may be applied by odontoblasts, which arise from the surrounding pulp tissue in response to the presence of the pulp stone; in addition fibrillar irregular calcified material may also be evident on the periphery of pulp stones [17].

Diffuse linear calcifications consist entirely of fine, fibrillar and irregular calcifications that develop in pulp chambers and canals. This material is often deposited in a linear fashion along the course of blood vessel or nerve [17].

\section{Primary Teeth and Pulp Stones}

In recent times, there have been many studies dealing with pulp stones in the deciduous dentition. One hundred and twenty primary maxillary and mandibular extracted teeth were collected to study the occurrence and nature of pulp calcifications by Kumar et al., [18] The teeth were serially sectioned, stained with hematoxylin and eosin, examined by light microscope. Pulp calcifications were observed in 31 $(25.8 \%)$ teeth. Two types of pulp calcifications were noticed i.e., diffuse calcifications and free/attached type denticles. They concluded that the low prevalence of pulp calcifications in the primary teeth supported the view that pulp calcification increases as the age advances.

Similar findings were stated by Yaacob and Hamid [19] who examined 120 primary teeth and found that only $6.7 \%$ of the teeth had 
pulp stones, with $11.7 \%$ having some diffuse calcification. Given that the size of the pulp stones ranged from 0.05 to $3.3 \mathrm{~mm}$, it is likely that the radiographic prevalence of pulp stones would be very low.

In contrast, Arys et al., [20] found that age did not have any influence on the occurrence of pulpal calcifications. They examined 42 primary molars and their results revealed that pulp stones were present in $78 \%$ of the molars, with $95 \%$ of the material showing some form of pulpal calcification. There was a lower incidence of pulp stones in treated and carious molars, which might be related to tertiary dentine formation or the low number of caries-free teeth. Further, in contrast to the Yaacob and Hamid [19] study, free or attached pulp stones were the most common type of calcification (as opposed to diffuse).

Holtgrave et al., [21] found a correlation between extensive pulp calcification and post-natally initiated fluoride prophylaxis, although the duration of tablet fluoridation (1-10 years) had no statistically significant influence on pulp calcification.

Thus even though there are contrasting findings regarding occurrence of pulpal calcifications in primary teeth, the dominant results conclude that the prevalence and complexity of pulpal calcification increases with age and has a large physiological component [22].

\section{Association with Systemic Conditions}

Pulp stones have been noted in patients with systemic or genetic disease such as dentin dysplasia, dentinogenesis imperfecta and in certain diseases such as Vander Woude syndrome [23].

There is also evidence that hypercalcemia, gout and renal lithiasis are pre-disposing factors to pulpal calcification [24].

Edds et al., suggested that $74 \%$ of patients with reported cardiovascular disease had detectable pulp stone, while only $39 \%$ of patients without a history of cardiovascular disease had pulp stones. This finding suggests the usefulness of dental radiographs to identify patients with cardiovascular disease for further screening and evaluation [25].

Bernick found calcification and lumen narrowing within extirpated dental pulp vessels, in both medium and small precapillary arteriole of cardiovascular patients [22].

Russell had investigated human pulp histologically on non-carious extracted teeth of seven patients suffering from diabetes for a longterm duration and control group of 13 non-diabetics. He concluded that calcification in angiopathies and thickened basement membrane were noted in both large and small blood vessels, and vascular changes seemed more pronounced in the central area of the pulp. Calcifications in diabetics were frequent and often sickle-shaped. In another histopathological study, conducted by Bissada and Sharawy's on 21 human dental pulps of diabetics and 20 matched controls, no vascular changes groups were found in the dental pulp of both. However, amorphous calcified bodies in the pulp of diabetics were found. Dental pulp of patients who suffer from diabetes mellitus tend to age more readily because it of obliterative endarteritis and because it has limited or no collateral blood circulation in fully developed teeth [26].

Seltzer demonstrated that attrition, abrasion, erosion and trauma could accelerate dental pulp complex aging changes. The circulation and innervations can rapidly become compromised, reparative dentin can be laid down at accelerated pace, and fibrosis and calcification can occur [27]. Protective pulpal response to irritation, such as secondary dentin formation, results in a decrease in the size of pulp chamber, and degenerative diffuse calcification might result in reduced ability to detect pulp stones in these teeth during radiographic examination [8].

\section{Clinical Significance}

The clinical significance of pulpal calcification is not completely understood. It has been reported upon numerous occasions that pulp stones are a cause of pain, varying from mild to severe excruciating pain [16]. Given the association between pulp stones and nerve tissue, both in terms of pulp stone formation and nerve fibre entrapment, it has been suggested that some pain of an idiopathic nature may be caused by pulp stones [28].

The consensus is that though denticles may seem to impinge on the nerves of the pulp, they probably do not. Therefore the extraction of teeth with radiographically demonstrable pulp stones in the hope of pain relief cannot be defended [16].

Along similar lines, pulp stones have been compared to kidney and gall bladder stones [29], but a much higher incidence of unexplained dental pain would be expected, given the high prevalence of pulp stones and pulp calcifications. Pulp stones have been described as symptoms of changes in the pulp tissue, rather than their cause [30].

The presence of pulp stones or diffuse calcifications does not affect the threshold of electric pulp testing [31].

However difficulty is frequently encountered in extirpating the pulp during root canal therapy if calcifications are present.

Attached stones may deflect or engage the tip of exploring instruments, preventing their easy passage down the canal [32]. Sometimes a large pulp stone can be dissected out of an access cavity using burs, but ultrasonic instrumentation with the use of special tips makes their removal far easier $[33,34]$.

Should a stone be attached to the canal wall and a file can be passed alongside the stone, it may be removed by careful instrumentation [35].

Generally speaking however, pulp stones present little clinical difficulty during root canal treatment when magnification, good access and appropriate instruments are employed.

\section{Conclusions}

Thus, pulp stones are mainly a sign of ageing of human pulp. They appear to be a part of normal physiological age changes in the body. This review has examined the various aspects of pulp stones including its types, etiology, incidence, relationship with primary dentition, correlation with systemic conditions and its clinical implications. However further research is still required, especially in the treatment point of view to enable increased success rate of endodontic therapy.

\section{References}

1. Bevelander G, Johnson PL (1956) Histogenesis and histochemistry of pulpal calcification. J Dent Res 35: 714-722.

2. Wilman W (1934) Calcifications in the pulp. Bur 34: 73.

3. Hill TJ. (1934) Pathology of dental pulp. J Am Dent Assoc 21: 820.

4. Orban's Oral Histology And Embryology. Editor: GS Kumar 12th edition page 128-129. 
Citation: $\quad$ Nupura Aniket Vibhute, Vibhute Aniket H, Daule RajendraT, Bansal Puja P and Mahalle Aditi (2016) Hard Facts about Stones: Pulpal

5. Gulsahi A, Cebeci AI, Ozden S (2009) A radiographic assessment of the prevalence of pulp stones in a group of Turkish dental patients. Int Endod J 42: 735-739.

6. Al-Hadi Hamasha A, Darwazeh A (1998) Prevalence of pulp stones in Jordanian adults. Oral Surg Oral Med Oral Pathol Oral Radiol Endod 86: 730-732.

7. Nayak M, Kumar J, Prasad LK (2010) A radiographic correlation between systemic disorders and pulp stones. Indian J Dent Res 21: 369-373.

8. Ranjitkar S, Taylor JA, Townsend GC (2002) A radiographic assessment of the prevalence of pulp stones in Australians. Aust Dent J 47: 36-40.

9. Moss-Salentijn L, Klyvert MH (1983) Epithelially induced denticles in the pulps of recently erupted, noncarious human premolars. J Endod 9: 554-560.

10. Baghdady VS, Ghose LJ, Nahoom HY (1988) Prevalence of pulp stones in a teenage Iraqi group. J Endod 14: 309-311.

11. Donta C, Kavvadia K, Panopoulos P, Douzgou S (2011) Generalized pulp stones: report of a case with 6-year follow-up. Int Endod J 44: 976-982.

12. Ozkalayci N, Zengin AZ, Turk SE, Sumer AP, Bulucu B, et al. (2011) Multiple pulp stones: a case report. Eur J Dent 5: 210-214.

13. Kretschmer OS, Seybold JW. (1936) The bacteriology of dental pulp stones. Dent Cosmos. 78: 292.

14. Stafne EC, Szabo SE (1933) The significance of pulp nodules. Dent Cosmos 75: 160.

15. Sundell JR, Stanley HR, White CL (1968) The relationship of coronal pulp stone formation to experimental operative procedures. Oral Surg Oral Med Oral Pathol 25: 579-589.

16. Shafer, Hine, Levy. Shafer's Textbook Of Oral Pathology. In: Rajendra R, Sivapathasundharam B (eds.) (6thedn) 578-579.

17. Neville, Damm, Allen, Bouquot. Oral And Maxillofacial Pathology. 3rd edition. page: 126-127.

18. Kumar S, Chandra S, Jaiswal JN (1990) Pulp calcifications in primary teeth. J Endod 16: 218-220.

19. Yaacob HB, Hamid JA (1986) Pulpal calcifications in primary teeth: a light microscope study. J Pedod 10: 254-264.

20. Arys A, Philippart C, Dourov N (1993) Microradiography and light microscopy of mineralization in the pulp of undemineralized human primary molars. J Oral Pathol Med 22: 49-53.

21. Holtgrave EA, Hopfenmüller W, Ammar S (2001) Tablet fluoridation influences the calcification of primary tooth pulp. J Orofac Orthop 62 22-35.
22. Bernick S, Nedelman C (1975) Effect of aging on the human pulp. J Endod 1: 88-94.

23. Kantaputra PN, Sumitsawan Y, Schutte BC, Tochraeontanaphol C (2002) Vander Woude syndrome with sensorineural hearing loss large craniofacial sinuses, dental pulp stones, and minor limb anomalies: Report of four generations Thai family. Am J Med Genet 108: 275-80.

24. Sayegh FS, Reed AJ (1968) Calcification in the dental pulp. Oral Surg Oral Med Oral Pathol 25: 873-882.

25. Edds AC, Walden JE, Scheetz JP, Goldsmith LJ, Drisko CL, et al. (2005) Pilot study of correlation of pulp stones with cardiovascular disease. J Endod 31: 504-506.

26. Bender IB, Bender AB (2003) Diabetes mellitus and the dental pulp. J Endod 29: 383-389.

27. Morse DR (1991) Age-related changes of the dental pulp complex and their relationship to systemic aging. Oral Surg Oral Med Oral Pathol 72: 721-745.

28. Seltzer S, Bender IB (1984) The Dental Pulp, (3rdedn) Philadelphia, PA: J.B. Lippincott Company.

29. Martin AP (2002) A radiographic assessment of the prevalence of pulp stones. Aust Dent J 47: 355-356.

30. Moss-Salentijn L, Hendricks-Klyvert M (1988) Calcified structures in human dental pulps. J Endod 14: 184-189.

31. Moody AB, Browne RM, Robinson PP (1989) A comparison of monopolar and bipolar electrical stimuli and thermal stimuli in determining the vitality of human teeth. Arch Oral Biol 34: 701-705.

32. Pashley DH, Walton RE, Slavkin HC (2002) Histology and physiology of the dental pulp. In: Ingle JI, Bakland LK (eds.) Endodontics, (5thedn) Hamilton, ON, Canada: BC Decker Inc, 43-5.

33. Stamos DG, Haasch GC, Chenail B, Gerstein H (1985) Endosonics: clinical impressions. J Endod 11: 181-187.

34. Pitt Ford TR, Rhodes JS, Pitt Ford HE (2002) Endodontics ProblemSolving in Clinical Practice. London, UK: Martin Dunitz Ltd, 85.

35. Pitt Ford TR, Mitchell PJC (2004) Problems in endodontic treatment. In Pitt Ford TR, (ed.) Harty's Endodontics In Clinical Practice, (5thedn) Edinburgh: Wright, 241. 